dans l'organisation du contrôle, autrement dit : dans l'application des textes, - œuvre coûteuse et difficile - qu'un long chemin reste à parcourir. La loi du 2 juillet 1935 a toutefois rendu la tâche plus facile à cet égard.

Pour le beurre, l'article 17 du même décret de 1924 a renforcé la définition du produit "naturel " par la fixation d'un maximum d'humidité.

Enfin, les définitions des diverses variétés de fromages, contenues dans le décret du 20 octobre 1936, basé à la fois sur la loi du 1er août 1905 et sur la loi du 2 juillet 1935, reflètent les exigences auxquelles j'ai fait allusion ci-dessus.

Ainsi, notre réglementation sur les produits laitiers a évolué dans un sens qui ne peut que réjouir les hygiénistes. Et la même orientation peut être constatée pour les principales autres denrées alimentaires et boissons.

Les esprits préoccupés d'assurer aux consommateurs, dans ee pays, une alimentation rationnelle, auront, croyons-nous, dès à présent satisfaction, s'ils veulent bien se rappeler que les pouvoirs publics ne peuvent menacer de sanctions pénales que ceux qui contreviennent à des textes précis, en laissant aux théoriciens et aux vulgarisateurs, dans le domaine de la liberté, le soin de faire prévaloir ce qu'ils considèrent comme des vérités nouvelles.

\title{
LA VALEUR DU DOSAGE VOLUMÉTRIQUE AU MÉTANAL DE LA MATIÈRE ALBUMINOIDE DU LAIT
}

par

\section{B. VAN DER BURG ET L. HABERS.}

Laboratoire de laiterie de l'Université agronomique (Wageningue), Pays-Bas.

Selon le décret royal néerlandais réglant le eommerce du lait, on détermine la teneur en matière albuminoïde du lait :

a) Par le dosage volumétrique au métanal, ou

b) par calcul, en multipliant la teneur en nitrogène d'après la méthode de Kjeldahl, par une constante.

Evidemment le législateur néerlandais considère ces deux méthodes de dosage de la matière albuminoïde du lait comme étant de même valeur.

Une recherche exécutée par l'un de nous (H.) en vue d'élaborer une méthode volumétrique convenable pour le dosage de la teneur en caséine du lait, nous fournit des données, qui nous portèrent à mettre en doute l'exactitude du dosage volumétriqué au métanal de la matière albuminoïde du lait. 
Il nous sembla utile d'étudier cette question de plus près. En ceci, nous sommes partis de la supposition que la méthode de Kjeldahl donne des résultats exacts pour la détermination de la teneur du lait en nitrogène.

En avril 1935, nous avons déterminé la teneur en nitrogène de 29 échantillons de lait, provenant chaque fois d'une seule vache et de là, calculé le taux en matière albuminoïde, $\mathrm{A}$, par multiplication par la constante 6,37 .

On détermina, dans les mêmes échantillons, le chiffre métanal, CM, o'est-à-dire la différence entre les quantités de soude caustique déci-normale, nécessaires à la neutralisation de $100 \mathrm{~cm}^{3}$ de lait avant et après l'addition de métanal en excès, en présence de phénolphtaléine comme indicateur. On calcula ensuite, pour chaque échantillon, la valeur du quotient A : CM.

Les dosages volumétriques au métanal ainsi que les déterminations de nitrogène ont été effectuées en double. Par la suite, nous avons fait usage des moyennes de ces deux déterminations. On les trouvera dans le tableau I. La moyenne générale de toutes les moyennes individuelles est 0,449 .

Pour déterminer la teneur en matière albuminoïde d'un lait, il suffirait donc, en cas de validité de cette méthode, de multiplier le CM par la constante 0,449 . Ce dernier chiffre diffère de $7 \%$ de la constante communément employée et recommandée officiellement.

Cette différence s'explique peut-être par le fait que nous n'avons employé dans nos recherches que du lait individuel et que ces recherches n'ont porté que sur un nombre assez restreint d'échantillons et cela dans les limites de temps très rapprochées d'un mois. On conçoit que le rapport entre les diverses combinaisons nitrogéniques du lait varie et que la saison influe sur cette fluctuation.

Nous avons démontré précédemment [1] que la caséine et la lactalbumine n'ont pas le même CM. Nous avons trouvé pour la caséine 0,475 et 0,379 pour l'albumine.

Pyne [2], dans une publication sur le dosage volumétrique au métanal de la matière albuminoïde du lait, démontre en effet une influence de la saison sur le chiffre nommé par nous CM.

Il n'y a donc pas lieu de s'étonner que nous ayons trouvé un facteur s'écartant assez sensiblement du chiffre officiel.

Si les recherches s'étendent sur une période plus grande, par exemple une année, et si l'on opère avec du lait de mélange, une conformité plus grande avec le chiffre officiel peut être attendue. En 1927-1928 nous avons recherché les chiffres correspondant à 36 échantillons de lait mélangé, représentant la production journalière d'une contrée desservie par une même laiterie. Le facteur trouvé fut 
TABLEAU I.

\begin{tabular}{|c|c|c|c|}
\hline No de l'échantillon & A & CM & $\frac{\mathrm{A}}{\mathrm{CM}}$ \\
\hline $1 \ldots \ldots \ldots \ldots \ldots \ldots \ldots$ & 2,74 & 6,60 & 0,415 \\
\hline $2 \ldots \ldots \ldots \ldots \ldots \ldots \ldots \ldots \ldots$ & 2,78 & 6,05 & 0,460 \\
\hline $3 \ldots \ldots \ldots \ldots \ldots \ldots \ldots \ldots$ & 2,66 & 5,75 & 0,463 \\
\hline $4 \ldots \ldots \ldots \ldots \ldots \ldots \ldots \ldots \ldots \ldots$ & 3,03 & 6,20 & 0,489 \\
\hline $5 \ldots \ldots \ldots \ldots \ldots \ldots \ldots \ldots$ & 2,97 & 6,80 & 0,437 \\
\hline $6 \ldots \ldots \ldots \ldots \ldots \ldots \ldots$ & 3,06 & 6,40 & 0,478 \\
\hline $7 \ldots \ldots \ldots \ldots \ldots \ldots \ldots$ & 3,36 & 7,85 & 0,428 \\
\hline $8 \ldots \ldots \ldots \ldots \ldots \ldots \ldots \ldots \ldots \ldots \ldots$ & 2,71 & 6,00 & 0,452 \\
\hline $9 \ldots \ldots \ldots \ldots \ldots \ldots \ldots$ & 2,46 & 5,75 & 0,428 \\
\hline $10 \ldots \ldots \ldots \ldots \ldots \ldots \ldots \ldots \ldots \ldots \ldots \ldots \ldots \ldots$ & 3,04 & 6,70 & 0,454 \\
\hline $11 \ldots \ldots \ldots \ldots \ldots \ldots \ldots$ & 2,62 & 6,45 & 0,406 \\
\hline $12 \ldots \ldots \ldots \ldots \ldots \ldots \ldots \ldots \ldots \ldots \ldots$ & 2,81 & 6,30 & 0,446 \\
\hline $13 \ldots \ldots \ldots \ldots \ldots \ldots \ldots \ldots$ & 2,78 & 6,90 & 0,403 \\
\hline $14 \ldots \ldots \ldots \ldots \ldots \ldots \ldots \ldots \ldots \ldots \ldots \ldots \ldots$ & 2,77 & 6,10 & 0,454 \\
\hline $15 \ldots \ldots \ldots \ldots \ldots \ldots \ldots \ldots \ldots \ldots \ldots$ & 2,72 & 6,30 & 0,432 \\
\hline $16 \ldots \ldots \ldots \ldots \ldots \ldots \ldots \ldots \ldots \ldots$ & 3,37 & 6,95 & 0,485 \\
\hline $17 \ldots \ldots \ldots \ldots \ldots \ldots \ldots \ldots$ & 2,98 & 6,65 & 0,448 \\
\hline $18 \ldots \ldots \ldots \ldots \ldots \ldots \ldots \ldots \ldots \ldots \ldots \ldots \ldots$ & 2,79 & 6,40 & 0,436 \\
\hline $19 \ldots \ldots \ldots \ldots \ldots \ldots \ldots \ldots \ldots$ & 2,50 & 5,70 & 0,439 \\
\hline $20 \ldots \ldots \ldots \ldots \ldots \ldots \ldots \ldots$ & 2,74 & 6,05 & 0,453 \\
\hline 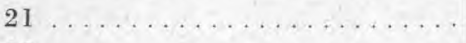 & 2,85 & 6,15 & 0,463 \\
\hline 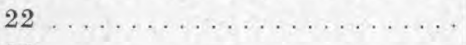 & 2,75 & 5,90 & 0,466 \\
\hline$\ldots \ldots \ldots \ldots \ldots \ldots \ldots$ & 2,82 & 6,65 & 0,424 \\
\hline $24 \ldots \ldots \ldots \ldots \ldots \ldots \ldots$ & 2,60 & 5,60 & 0,464 \\
\hline 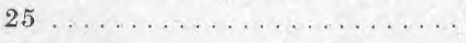 & 3,04 & 6,75 & 0,450 \\
\hline $26 \ldots \ldots \ldots \ldots \ldots \ldots$ & 2,93 & 6,40 & 0,458 \\
\hline $27 \ldots \ldots \ldots \ldots \ldots \ldots \ldots \ldots \ldots$ & 2,33 & 5,35 & 0,436 \\
\hline 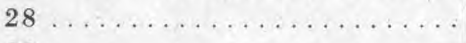 & 3,26 & 6,55 & 0,498 \\
\hline $29 \ldots \ldots \ldots \ldots \ldots \ldots \ldots \ldots \ldots$ & 2,86 & 6,10 & 0,469 \\
\hline
\end{tabular}

$$
\text { Moyenne : } \mathrm{A}=2,84 \quad \mathrm{CM}=6,32 \quad \frac{\mathrm{A}}{\mathrm{CM}}=0,449
$$

$$
\text { Erreur moyenne de }: \frac{A}{C M}= \pm 0,023
$$

alors 0,486 ; l'écart du chiffre officiel n'était plus que $0,8 \%$. Pour les valeurs particulières on peut consulter le tableau II.

Une telle conformité des valeurs moyennes ne prouve cependant pas encore la valeur de cette moyenne pour le calcul de la teneur en matière albuminoïde d'un échantillon quelconque de lait. Pour pouvoir juger de la valeur pratique d'une moyenne, il nous faut considérer comment cette moyenne a été obtenue. 
TABLEAU II.

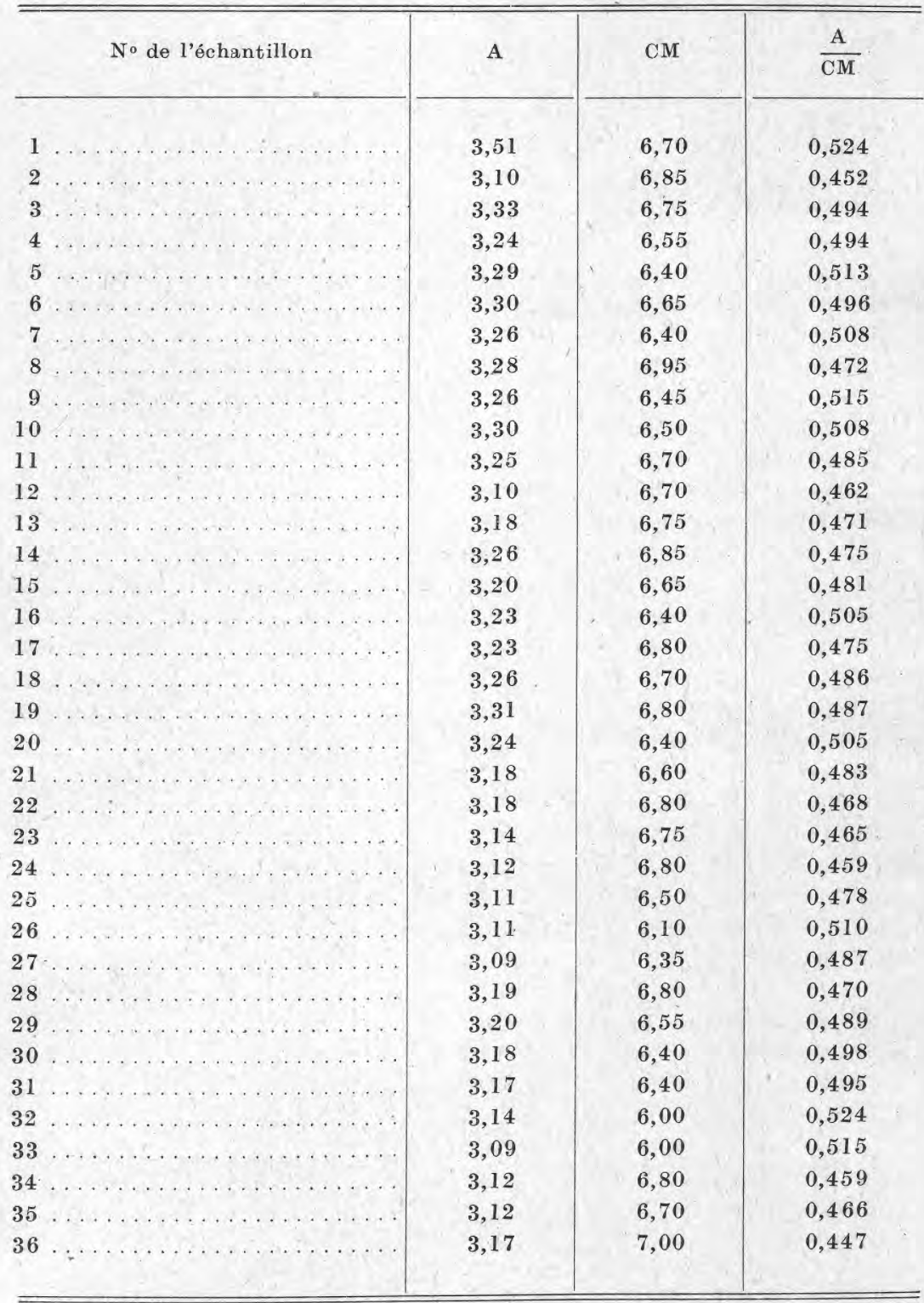

Moyenne : $\quad \mathrm{A}=3,20 \quad \mathrm{CM}=6,60 \quad \frac{\mathrm{A}}{\mathrm{CM}}=0,486$

Erreur moyenne : de $\frac{\mathrm{A}}{\mathrm{CM}}= \pm 0,021$ 
Voyons d'abord les données fournies par l'examen de lait de vaches prises individuellement.

Comme quotient A : CM nous avons trouvé des valeurs intermédiaires de 0,403 et 0,498 . Caleulons, en nous basant sur les données du tableau I, l'erreur moyenne d'une seule observation selon la formule $F= \pm \sqrt{\frac{\sum \mathrm{f}^{2}}{\mathrm{n}-1}}$

Dans cette formule $f$ représente la différence entre une seule observation et la moyenne arithmétique de toutes les observations et $n$ désignera le nombre total d'observations. Le chiffre trouvé est $\pm 0,023$. On peut alors calculer que les limites extrêmes entre lesquelles évoluera, avec la très grande probabilité de $99 \%$, la valeur du facteur A : CM, seront : 0,380 et 0,518 . Ces chiffres s'obtiennent en diminuant et en augmentant la moyenne du triple de F. Ainsi un $\mathrm{CM}$ de 6,3 donne une teneur en matière albuminoïde de $6,3 \times$ $0,499=2,83 \%$, valeur qui cependant accuse une très grande incertitude, du fait que dans les cas extrêmes elle pourrait être $6,3 \times$ $0,380=2,39 \%$ ou $6,3 \times 0,518=3,26 \%$.

Il nous reste encore à rechercher si le matériel du tableau II (lait mélangé, échantillons prélevés sur l'année entière) donne peut-être une autre image de la valeur du dosage volumétrique au métanal de la matière albuminoïde du lait. Nous savons que le chiffre moyen trouvé comme A : CM est 0,486 . Les limites de 36 valeurs étaient 0,446 et 0,524 . L'erreur moyenne d'une seule observation est ici $\pm 0,021$, d'où il suit que les limites extrêmes du A : CM sont : 0,423 et 0,549 . Ceci donne, pour la teneur du lait en matière albuminoïde, avec un $\mathrm{CM}$ moyen (en l'occurrence 6,6 ), une valeur de $6,6 \times 0,486=3,21 \%$ avec la réserve que cette teneur pourrait également être $6,6 \times 0,423=2,79$ ou $6,6 \times 0,549=3,62 \%$.

Nous en concluons done que :

La détermination de la teneur du lait en matière albuminoïde par le dosage volumétrique au métanal n'a pas l'exactitude exigible.

\section{BIBLIOGRAPHIE.}

[1] B. VAN DER BURG. Off. Org. v. d. Alg. Ned. Zuivelbond, 23, 1928, p. 330-333.

[2] G. T. Pyne. Biochem. Journal, 27, 1933, p. 915-917. 